\title{
VISUALISATION OF COMMUNICATION IN COLLABORATIVE VIRTUAL ENVIRONMENTS
}

\author{
Kirsi Heiskanen ${ }^{1}$, Mikko Jäkälä ${ }^{2}$ and Samuli Pekkola ${ }^{2}$ \\ 1) Kuopio Vocational Adult Education Centre, Kuopio, Finland \\ 2) Department of Computer Science and Information Technology, University of Jyväskylä, \\ Finland
}

\begin{abstract}
Human communication is a multifaceted and ambiguous process, and even more so when it takes place in innovative technical environments. In this paper, advantages and disadvantages of virtual reality as a communication medium are discussed from the viewpoint of the visualisation of communication. Embodiments of human beings are analysed and some basic requirements for designing appropriate representations are suggested.
\end{abstract}

\section{INTRODUCTION}

It is a challenge to model and visualise human communication in technical environments, in this case Virtual Reality (VR). The term 'virtual reality' is understood differently from person to person. A traditional and widely accepted interpretation is that VR is an artificial environment designed to model reality by using one or more computers. VR can also be understood as an alternative way to stimulate different senses of a human being, or to create a feeling of being in some other place than where the user is physically.

VR systems which are used to support co-operation and collaboration between different users, are usually called 'Collaborative Virtual Environments' (CVE). Typically these systems run on desktop computers, and no other special devices are needed. Since there are no advanced input and output devices attached to the system, the user expectations lie heavily on the visualisation of communication on the computer screen.

Within the communication process, different symbols and codes are being used in order to transfer meanings from one communicator to another. During the communication process, two or more communicators work towards a shared meaning and understanding of a certain, often abstract, matter. Human communication can roughly be divided into two categories: verbal and non-verbal communication. Verbal communication is often transmitted through spoken or written language. However, human communication is most effective when both verbal and non-verbal channels are used. Especially, non-verbal communication and its rich nuances can 
enhance interaction between participants in certain situations. There have been several different attempts to visualise non-verbal communication. Despite these attempts, it remains the most challenging area in designing communicative CVE applications.

In this paper, collaborative virtual environments are discussed from the communicative point of view. What are the essential features of human communication which should be taken into consideration in order to create effective and appropriate communication spaces? In the first part of the paper the different characteristics and features of human communication are discussed, while the second part concentrates on the visualisation of human communication in collaborative virtual environments. Finally, some basic requirements for a communicative CVE are presented.

\section{HUMAN COMMUNICATION}

Effective communication is based on the communication skills of the participants. Some skills are suitable for all situations and audiences, whereas some skills are context-based. In this paper, human communication is discussed in order to highlight those factors of communication that hold importance in the context of virtual reality applications.

If communication process is approached in a more systematic way, some general characteristics of communication can be found. However, these characteristics are just some aspects of human communication, since the term itself is not very clearly defined. Communication can be determined as symbolic behaviour. This means that there is an agreed relationship between an object in real life and a certain symbol. The most familiar example of symbolic behaviour is the use of language. Each language is a code which allows its users to transform ideas and thoughts into a meaningful messages and utterances. The key idea is the arbitrary relationship between the symbol and the object it represents.

A shared code is the basis of symbol-meaning relationships. The more similar the code that the participants are sharing, the more probable is the similarity of meaning. It is often assumed that when people share language as a code, they also share the meanings for the symbols they use. Sharing a code is not that simple. For example, if English, American English and World English are compared, there are several words that have different meanings, e.g. in England or in the United States. Therefore people negotiate and create meanings in the course of interaction.

Intentionality in communication is the level of consciousness or purposefulness of a communicator in encoding messages. Some communication scholars state that communication takes place only when a message is intentionally constructed and transmitted (O'Hair et al. 1996). Yet, for instance, emotional displays are easily read across cultures and they are often not intentional. One cannot help oneself from blushing, etc. 
However, these unintentional emotional displays are communicative in everyday usage because there is a consensus about their meaning. A person knows what blushing means in a given situation. In practice we usually tend to see a person as more credible when she consciously or purposefully gives information to somebody else in comparison to when the other just gleans information from observation or overhearing. However, unintentional information is often evaluated as more honest and sincere, since the other person does not have the opportunity to censor or modify it. Although some messages transmitted through the emotional communication system are highly reliable and easily interpreted, most of the messages are ambiguous and open to different interpretations. Most of these kind of messages can be interpreted only on the based of contextual cues, and the validity of interpretation is often uncertain.

Communication requires a medium to transport the symbols. Face-to-face communication is often seen as a prototype of communication. With new communication technologies, distinctions between face-to-face and other methods of communication are becoming more problematic. Selecting the appropriate media will become increasingly important while the number and complexity of available media increases. When selecting the media, the criterion might be the immediacy and interactivity it offers. The use of the telephone as a medium is totally different from the use of e-mail or traditional letter.

Communication is a transactional process. This means that at least two people must be involved in symbolic exchange for communication to occur. In communication, the speaker always attempts to influence the listener in some way. At the same time, the speaker himself or herself is open to be influenced by others. The transactional process of communication is not only a static exchange of symbols but also a dynamic process based on the communication behaviour of the participants. Therefore all the participants are responsible for the outcome and whether the goals met.

One more characteristic for communication is cultural boundaries. Communication is difficult to separate from culture because they both are language bound. However, the relationship between communication and culture goes far beyond obvious language differences. Culture has influence on what is named in the environment, how fine the linguistic distinctions are, and how language is used to interpret the world around people. Even within a single country where people speak the same language, some cultural boundary problems may occur. Subcultural, co-cultural and ethnic differences can lead to major differences in the way people use and interpret language and non-verbal behaviour. For example, an utterance that counts as an insult in one subculture can be considered as a compliment in another (O'Hair et al 1996). 


\section{VERBAL AND WRITTEN COMMUNICATION}

The characteristics of human communication create a multifaceted and even a complex scenario, which is affected by the communicators' level of knowledge, earlier experiences, attitudes, values and beliefs. Also the message, media and code, the competence of both speaker and listener, as well as the feedback, play vitally important part in this process (Ehninger et al. 1986). Sometimes it might be difficult to translate meaning into the code of spoken language when so many factors are involved. Meanings seem to be the core of human communication. They are not in words, but in people who use words in order to approximate the meaning to be communicated. Words are relatively stable but meanings are indefinite in number and changing all the time, as well as new meanings being given to words.

According to DeVito (1991) words are only a poor substitute for complex feelings and thoughts. That is why words can only approximate intended meanings. Language and words within a certain culture represent only a part of our communication system. Coding meanings into verbal form is difficult, because they can have different denotative and connotative meanings and they can also be represented in different levels of abstraction. Therefore effective integration of both language and non-verbal signals is vitally important in real communication.

Spoken language is often seen as more spontaneous than written text. Lots of time can be spent on formatting certain matter into a fluent text. Text can be revised several times, and the text the audience reads may be very different than the first draft version. Also the interpretation of the text varies from person to person and from time to time. If we make mistakes when we are communicating with the aid of spoken language, it is easy to correct parts if the speaker or the listener notices them. Immediate feedback is the main advantage of face-to-face communication. If the ideas or thoughts are expressed as text, it is possible that there will be no feedback at all or at least it will reach the presenter after the text has been composed. However, new media will mix these features. For example, when chatting in a textbased environment, the outcome is spontaneous and not polished like written language often is. The language has a written form but it is very close to spoken language.

\section{NON-VERBAL COMMUNICATION}

The term non-verbal communication is used to refer to a process of signalling meaning through behaviour which does not involve the content of spoken words. Non-verbal communication refers to intentional and unintentional messages which are encoded and decoded with meaning. They also serve specific functions since behaviour is seen as attributed meaning. Because of the fact that almost every behaviour can be interpreted as having meaning, the range of non-verbal communication is very wide. 
Non-verbal behaviour is often a better indicator of thoughts, feelings and emotions than spoken or written language. From the social point of view, non-verbal communication gives clues that can be used to interpret messages and meanings embedded in them. If there is a contradiction between the verbal and non-verbal communication, the listener often relies more on the non-verbal message since it is more spontaneous and carries more true feelings than the controlled spoken language.

Non-verbal communication can be divided into types of code that produce potential messages. These codes are the means by which messages are sent and received. According to Patton and Griffin (1977), Rosenfeld and Berko (1990), DeVito (1991) and O'Hair et al. (1996) non-verbal communication can be classified into following types: appearance and artifacts, kinesics, facial expressions, oculesics, paralanguage, haptics, proxemics, chronemics and olfactics.

Appearance and artifacts are often a basis for judgements. Most people are aware of the significance of attractiveness and therefore a lot of money is spent on fashionable clothes, jewellery, makeup, haircuts, etc. There is a good reason for that. Attractive people seem to get more attention, which means more possibilities to interact with others and, for example, have a better chance of getting a job. The evaluation of appearance is strongly culture-bound and does not only influence perceptions of attractiveness but also evaluations of the individual's background, character, personality, status and predicted behaviour. Evaluations are based on body shape and size, facial features, skin colour, as well as clothing including different accessories.

Kinesics is a term for the communicative ability of gestures and body movements. It can be classified into five categories of kinesic behaviour: emblems, illustrators, regulators, adapters and affect displays. Emblems are movements and gestures that have a direct verbal translation in a certain culture or within a group of people. Emblems are intentionally used to replace words when they offer more effective ways of communication than spoken language (an often used example of emblems is the hitchhiker's thumb or a wave of the hand to greet another person). Illustrators are movements or gestures that are used to illustrate or accompany a verbal message. Usually illustrators can not be interpreted without the use of words. Regulators are used to regulate conversation, e.g. by raising a hand or leaning back. Adapters are behaviours exhibited to satisfy a physical or psychosocial need, such as rubbing eyes when tired or biting nails when nervous. Affect displays are used to indicate emotional or affective state. These are often unintentional movements that reflect the sender's true emotions and thereby convey moods and feelings.

Facial expressions are primarily used to display emotions. Some of the emotional states are clearly apparent while some are more difficult to interpret. The most researched and most common facial expression is the smile and its different variations. Facial expressions are often unintentional. 
However, some intentional control can be used. Intensification is a facial management technique, which is used to exaggerate what is felt: it is the opposite of deintensification which is used for downplaying. Neutralisation means elimination of all expressions of emotion. Masking is used to replace the expression of true feelings with expressions which are deemed appropriate for that particular situation.

Oculesics refers to eye behaviour. Eye behaviour has six communicative functions. Eyes can influence attitude change and persuasion, indicate degrees of attentiveness, interest and arousal, express emotions, regulate interaction, indicate power and status, as well as form impressions in others. Eye behaviour varies from mutual gaze to gaze omission. It can also be interpreted as eye contact indicating involvement in interaction or as staring - eye behaviour with negative connotations.

Paralanguage carries the communicative value of vocal behaviour - not what is said but how something is said. Paralanguage can convey feelings and emotions as well as personal characteristics and attraction on the basis of vocal cues. These cues can be used in the interpretation of spoken language, and the meaning behind the words can be found. There are some general features of paralanguage: voice qualities (tempo, resonance, rhythm, articulation, and pitch range) and vocalisation (a vocal cue that does not have the structure of language). Vocalisations can be further divided into three classes: vocal characteriser (that is a sound that conveys the emotional or physical state of the speaker), vocal qualifier (which qualifies or regulates a verbal message) and vocal segregate (that is a sound which has a connotative meaning).

Haptics or tactile communication refers to touching behaviour. Touching other people has very strict cultural and social norms and rules. Haptics is often seen as one of the most fundamental types of communication from a private kiss to a public handshake - from love-intimacy to functionalprofessional touch.

Proxemics means the communicative aspects of the use of space. One area of proxemics is personal space around one's body to which one attaches ownership. Hall (1959) devised an often referred to system of identification for the spatial zones according to the type of interpersonal relationship. These zones vary from intimate zone to public zone. The use of space is culture bound and it can convey, for example, power, status and territoriality.

Chronemics means the communicative ability of the use of time. The use of time and the feelings about time send strong non-verbal messages. So chronemics refers to how people perceive and structure time. In some cultures time is valued and in some others time is seen as a less important factor. Differences in orientation can create communication problems since it affects the importance that person ascribes to conversation content, the length and urgency of interaction as well as punctuality. 
Olfactics refers to the communicative characteristics of smells. Although smell is one of the primary senses, it is the least researched code of nonverbal communication. Smells can produce strong reactions and intensify positive or negative perceptions. Offensive odours will increase distance between communicators and shorten the time spent in interaction; likewise pleasant odours will increase communication with others. Attractiveness is also often associated with scent, which may determine whether an interaction is initiated, continued or terminated. Cultural norms often provide guidelines to distinguish between offensive and pleasant smells.

Human communication consists of various different verbal and non-verbal aspects as was shown earlier. Therefore human communication may sometimes be extremely difficult even though it could take place in a faceto-face context. In the next section the context is provided by a new media, virtual reality. It will give new possibilities for communication by combining several media. Simultaneously some new challenges are introduced.

\section{COMMUNICATIVE APPROACH TO CVE}

Currently, many CVE applications attempt to support features of human behaviour. However, they lack many aspects of natural communication thus a feeling of real communication cannot be easily achieved. Technical problems, limitations and errors result in changes in the quality of the connection causing the communication process to become even more inconvenient.

There is a clear attempt to imitate most features of human communication in VR applications. For example, appearance, kinesics, proxemics, facial expressions, and oculesics are all to some extent included in avatars. However, this loads avatars with lots of information. Very often this information is not clearly or unambiguously visualised, in some cases it is not visualised at all. This gives reason for a critical examination of those features of human communication that should, or should not, be included in avatars.

Haptics or tactile communication is used in some applications to activate actions or objects. For example, in DIVE (Carlsson and Hagsand 1993) touching, or actually crashing against other objects, opens a channel for communication (e.g. a portal or a gateway, or even some other application). Olfactics is seldom used in VR applications, since one may argue that it is not an important code of communication. However, there are some cases in which scents could be important in giving information, but this information could be given technically more easily with other codes (Krueger 1996). Chronemics is a basic feature of the general behaviour of human beings it affects communication in every level. Thereby it is included in every action that the user makes. If the other participants are forced to wait for the other communicator to interact, they would probably behave as in reality. They could be annoyed with the wait, they could lose their interest and 
concentrate on other activities, or they could simply ask what is going on. Chronemics can also be included in the communication process if the system manages the usage of time of the users to some extent. This may create different responses among the users.

If avatars and an environment represent most of the information concerning human communication, it will burden the users visually. If the application is very rich in details, this may overload the user and he or she cannot concentrate on all information available. Therefore, e.g. 3D sounds can be used to focus the attention of the user on certain events. Also, if it is technically possible, the quality of voice connection should be improved, so paralanguage could be used efficiently in communication. Simultaneous use of different codes could clarify messages. If the users are able to hear and see the information, the interpretation of the messages into meanings is easier to perform.

When the user is able to receive and interpret different messages, a feeling of being somewhere else than the place where the user is physically located could be achieved. This kind of feeling is hard to create, so a combination of several different media and communication tools are needed. Hollan and Stornetta (1992) argue that simulating face-to-face co-presence is a bad design objective. Each medium has its own advantages and disadvantages, and a combination of several different media is needed to achieve a feeling of really being somewhere else.

\section{AVATARS AS REPRESENTATIONS OF PEOPLE}

Representing people in collaborative virtual environments is essential in order to provide 'naturalistic', a natural platform for communication and group work. As in reality, the embodiment of the user, i.e. the avatar, reflects many aspects from the user. However, as the following quote indicates, the same limitations of appearance as apply in reality does not necessarily have to exist.

"Your avatar can look any way you want it to, up to the limitations of your equipment. If you're ugly, you can make your avatar beautiful. If you've just gotten out of bed, your avatar can still be wearing beautiful clothes and professionally applied makeup. You can look like a gorilla or a dragon or a giant talking penis in the Metaverse. ..."

Neal Stephenson, Snow Crash, pp. 33-34.

The appearance of a person is important in reality as has been shown earlier. However, what is the implication of it in VR? Because VR does not have to mirror reality, the user can choose his or her looks to represent whatever he/she wishes. This causes several difficulties and even problems for human communication, since the conventions of attractiveness do not apply. The avatar could be any size or colour, or it's shape may vary from a simple blockie to very complex creature (see Figure 1 for some examples). For 
example, an ugly person may take on a pretty appearance or vice versa, so no judgements based on the avatar's appearance can be made.

The user might even be an embodiment of something which is usually regarded as non-human or a static representation. The avatar might have the appearance of an animal or some strange object (e.g. a guitar, a mask, a logo). This causes even more problems, since culture-bound rules, norms and beliefs could be broken. It is extremely hard to make judgements of a user and her personality if she, for example, has an appearance of an apple. However, the choice of the avatar indicates several characteristics of the user. The apple-avatar might be chosen because of its pretty appearance, or because the user likes apples, or even because it was the only suitable (but not the exact) one she wanted to look like.
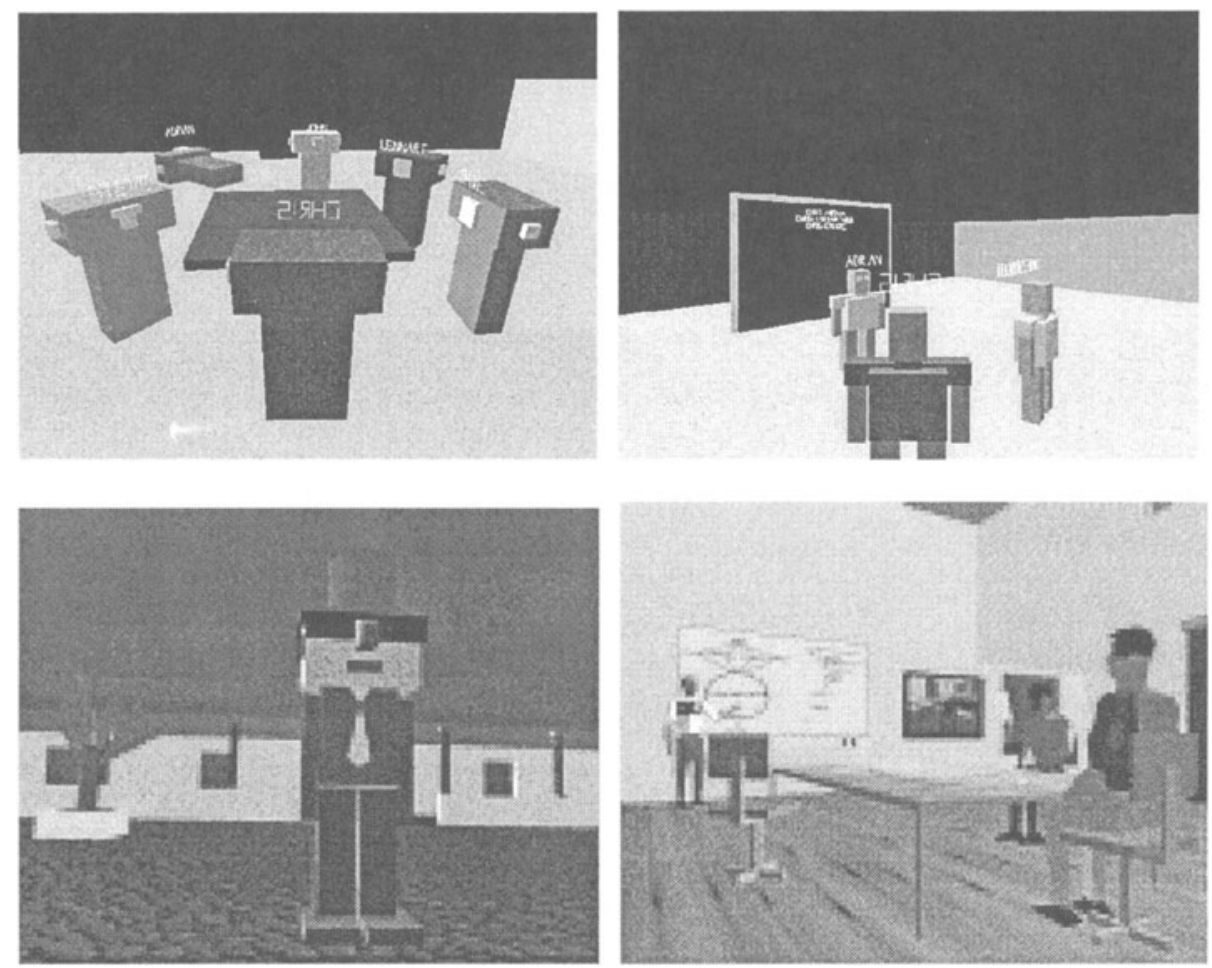

Figure 1. Different avatars. Top: MASSIVE (blockies and more developed avatars), bottom: DIVE (a blockie and virtual humans). ${ }^{l}$

This kind of anonymity provides some new alternatives for communication and collaboration. Because it is possible that other people do not have a slightest idea of the other's identity, it is much easier to discuss some sensitive topics. For example, research on workplace romances and sexual harassment is extremely sensitive, and the anonymity of both the 
interviewer and the interviewee eases the situation (Pierce and Aguinis 1997). However, it is not necessary to gain anonymity by choosing a very strange looking avatar. It is more natural to communicate with a person rather than with an apple. A talking apple-avatar might even confuse an inexperienced user and create some fears to express one's own feelings and thoughts.

Kinesics eases communication in VR, since other people are able to see some direct connections to human communication in reality. A talking apple with gesturing hands lowers the threshold to enter the conversation and exchange ideas, despite the unfamiliar appearance of the avatar. However, it is not natural that an apple is equipped with hands, so the inexperienced user may focus only on subsidiary issues, which may even complicate attempts at communication rather than aiding it. The importance of hands can be questioned. The situations when the hands are actually needed are the moments when the user points in some direction or at some object, or when some objects are attached to the avatar, i.e. the user is carrying something along with her. Of course, there are several situations when a hand (or some other indicator) is needed to express one's feelings and thoughts. For instance, if the user wishes to greet someone, she may wave her hand. On the other hand, waving the hand is a culture-bound issue, so greeting can be performed also by bowing, rubbing noses, or by shaking hands accompanied with a bear hug (O'Hair et al. 1996).

Body movements and the use of space in VR express similar issues as they do in reality. Bowers, Pycock and O'Brien (1996) showed that participants move their avatars around to get a better view of the environment and of those they wish to interact with or pay attention to. However, since the number of movements of an avatar is limited, turns and movements might create misunderstandings to others. Many applications do not separate head movements from the body movements, so the only way to glance is by changing the whole body orientation. As Bowers et al. (1996) suggested, and we agree, the introduction of joints to the embodiments seems to be worthwhile in order to distinguish 'looking-around' from 'turning-away'.

Boundaries of space in VR do not have to have the same meaning as they do in reality, e.g. the walls could be transparent and permeable. However, many meanings are the same. Walls and other boundaries are used to separate different sections of the environment. The division could be based on environmental (walls, objects) or social (people, groups) aspects of the community. Different objects are used to aid people in forming subspaces and subgroups. For example, a table forms a natural platform for (private) group conversation while a podium can be used for expressing one's thoughts to everyone. Technically, interaction and conversation can be managed by changing the size and shape of the aura, and some variables which specify the level of awareness within a certain region ${ }^{2}$. On a table metaphor, aura covers only the participants around the table. On the other hand, at the podium every participant within a certain area is being covered. However, it is possible that the aura is different for each media. For example 
a person is able to see another but cannot hear him or her, so by moving closer an invisible boundary is passed and voice connection is activated. This movement expresses the person's interest in such event to everyone, as it does in reality.

In general, the use of space and movement are difficult topics in CVEs. The use of navigation devices is not very natural; for instance, simultaneous talking and walking is rarely performed. During the conversation, movements usually consists of only looking around to find out what is going on, and some 'step-ups' as introduction movements (Bowers et al. 1996). Our experiences are similar.

Maybe the most difficult task in visualising communication is the question of displaying emotions and facial expressions. Facial expressions are hard to convert to an avatar, not only technically but also socially, since the input devices are not sufficiently advanced. The most suitable medium for capturing emotions from one's face is, in most cases, video. However, there are some technical requirements which limit the use of it, e.g. huge bandwidth requirements on the transmission channel, the computer the receiver uses is not powerful enough and cannot display video, or simply the transmitting person does not have a video camera.

Therefore, some alternatives for visualising communication have been developed. For example, there might be a tool bar, where the user is able to choose different emotions to the others (see Figure 2). However, this kind of scheme is not natural behaviour, since the user must perform several additional actions to display her emotions, i.e. one to start smiling and another to stop it. If either of these actions is missing, the emotion is retained on the face and a great possibility of misunderstanding exists. Similar problems occur with any device which requires additional actions to show emotions on the avatar.

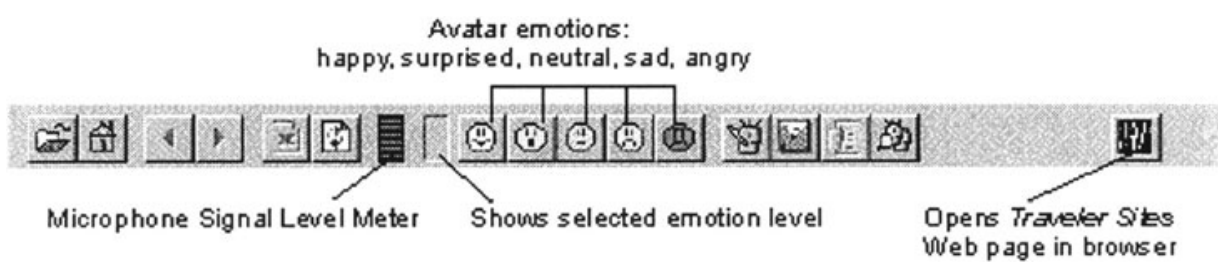

Figure 2. Onlive! Traveller ${ }^{3}$ tool bar for showing emotions.

This analysis leads us back to the use of video. If it is captured from one's face and then reconstructed in VR, there is no need for additional actions taken by the user. It is only required that the user stays at the front of the video camera and performs her actions there without making too large movements. In VR, a created image could be a direct video stream (like a TV) or a virtual face, which is reconstructed by the data shot by the camera (Achermann, Jiang and Binke 1997, Proesmans and Gool 1997). The former requires a lot of bandwidth from the network and the latter burdens the computers, so it is difficult to achieve satisfactory picture quality (Pekkola 
and Robinson 1997). An image with bad quality and slow animations is not very informative, so its added-value to human communication won't be very big either.

Even very low picture quality bears some information about eye and mouth movements. Because avatars cannot glance (in the sense human beings are able to), eye-contacts and gazes can only be achieved by getting the information from the user through a video. However, usually there is no camera mounted on the monitor, so it is extremely hard or even impossible to gain eye-contact. Instead of using the avatar's eyes for eye-to-eye contact, they can be used to indicate whether the user is actively using the application. E.g. wide-open or blinking eyes express that the user is behind his or her desk and really using the application, while closed eyes could indicate absence. The mouth and its movements can be used to indicate that someone is talking. Simple movements are enough to show that, even though lip reading is not possible.

The latest approach to representing people are virtual humans, i.e. avatars which look and behave like ordinary people. Because the appearance and behaviour are very humane, the communication becomes more natural with all its multiplicities.

\section{CONCLUSIONS}

The multiplicities and difficulties of normal, face-to-face communication are reflected in CVEs. Also, some new problems and challenges arise with the new media, and form even more complex problems to be solved. To achieve a feeling of being somewhere else, and communicating with other people in that environment, sets many requirements on avatars and on the environment. The environment does not directly influence the communication while the avatars are essential. Therefore some minimum requirements for the appearance and the functional features can be set. Some kind of embodiment must exist, but physical looks could be almost anything. However, some functional features are essential, i.e. a mouth, which indicates the talker, an eye, which is needed to show the orientation of an avatar, and a pointer to point out directions.

These are minimal requirements for basic communication. However, to make communication more natural many more features are needed. For example, video is essential to show facial expression, body joints to distinguish 'looking-around' from 'turning-away' and hands to express gestures. However, there is always the danger of decorating the avatar too much, and that way losing the information and codes behind all the (unnecessary) adornments. Altogether, every step towards natural appearance and behaviour makes it easier to understand human communication through virtual reality environment. 


\section{Acknowledgements}

We wish to thank the researcher, M.A. Peppi Taalas (University of Jyväskylä, Finland), for her valuable comments, proof reading and revisions.

\section{NOTES}

1 The pictures were originally presented at the systems developers' www-pages. See http://www.crg.cs.nott.ac.uk/research/systems/MASSIVE/screenshots/ and http://www.sics.se/dive/demos/images/

2 The spatial model of interaction (Benford and Fahlén 1993) defines a focus (person's level of awareness of other participants) and a nimbus (other participants' awareness of a certain person). Those variables can be used for managing the communication between the participants.

3 The picture was originally presented at http://www.onlive.com/tech/trav/travuq/travyg20.html

\section{REFERENCES}

Achermann, B., X. Jiang, H. Binke (1997) Face recognition using range images. Proceedings of Virtual Systems and Multimedia, VSMM'97, Geneva, Switzerland. IEEE Computer Society.

Benford, S. and L. Fahlén (1993) A Spatial model of interaction in large virtual environments. Third European Conference on Computer Supported Cooperative Work ECSCW'93. 13-17 Sept. Milan, Italy, Milan, Italy, Kluwer Academic Publishers.

Bowers, J., J. Pycock, J. O'Brien (1996) Talk and embodiment in collaborative virtual environments. Proceedings of $\mathrm{CHI}^{\prime} 96$, Vancouver, Canada, ACM Press.

Carlsson, C. and O. Hagsand (1993) DIVE - a platform for multi-user virtual environments. Computer \& Graphics 17(6) 663-669.

DeVito, J. A. (1991) Human communication. Harper Collins, New York.

Ehninger, D., B. E. Gronbeck, R. E. McKerrow and A. H. Monroe (1986) Principles and types of speech communication. Scott, Foresman and Company, Illinois.

Hall, E. (1959) The silent language. Doubleday, New York.

Hollan, J. and S. Stornetta (1992) Beyond being there. CHI '92: Striking a balance. ACM, Monteray, CA.

Krueger, M. W. (1996) Reality stinks: and so should virtual reality. International conference on Virtual Systems and Multimedia (VSMM'96), Gifu, Japan.

O'Hair, D., G. W. Friedrich, J. M. Wiemann and M. O. Wiemann (1996) Competent communication. St. Martin's Press, New York.

Patton, B. R. and K. Giffin (1977) Interpersonal communication in action. Harper \& Row, New York.

Pekkola, S. and M. Robinson (1997) Collaborative virtual environments, real-time video and networking. Proceedings of International Conference on Virtual Systems and Multimedia, VSMM'97, Geneva, Switzerland, IEEE. 
Pierce, C. A. and H. Aguinis (1997) Using virtual reality technology in organizational behaviour research. Journal of Organizational Behaviour 18 407-410.

Proesmans, M. and L. V. Gool (1997) Reading between the lines - a method for extracting dynamic 3D with texture. Proceedings of ACM Symposium on Virtual Reality Software and Technology 1997, VRST'97, Lausanne, Switzerland, ACM Press.

Rosenfeld, L. R. and R. M. Berko (1990) Communications with competence. Scott, Foresman/Little, Brown, Illinois. 